International Journal of Computer Networks \& Communications (IJCNC) Vol.3, No.3, May 2011

\title{
TOWARDS SERVICE SONTINUITY IN IMS HeTEROGENEOUS ACCESS NeTWORKS
}

\author{
Mohammed BOUTABIA and Hossam AFIFI \\ TELECOM SudParis, CNRS SAMOVAR UMR 5157 \\ 9, Rue Charles Fourier - 91011 Evry Cedex, France \\ \{mohamed.boutabia, Hossam.afifi\}@it-sudparis.eu
}

\begin{abstract}
In this paper we tackle the mobility issue in IP Multimedia Subsystem (IMS). Although IMS was designed to integrate different access networks, mobility management among these networks is still unresolved. We propose a novel hybrid mobility management scheme, based on tight cooperation between fast handovers for mobile IPv6 (FMIPv6) and session initiation protocol (SIP) to ensure an uninterrupted real-time service. Moreover, the new Media Independent Handover (MIH) service is integrated into IMS architecture in order to perform intelligent and accurate horizontal and vertical handovers. We investigate two handover cases: selected handover and forced handover. Selected handover takes place when user equipment (UE) is connected to the network via two interfaces at the same time and decides to upgrade the quality of its connection following a given criterion (i.e. cost, bandwidth...etc) without having any difficulty in the previous link. As far as forced handover is concerned, it occurs when the signal reaches a critical level and UE is forced to make a handover in order to maintain the ongoing communication. This case is managed in two phases. The first one or the fast phase is handled by FMIPv6 protocol to preserve as soon as possible packets of the ongoing communication. The second one or the slow phase is handled by SIP protocol to optimize packet delivery route. By doing so, we exploit the benefits of both network layer and application mobility protocols to ensure a continuous session over the two networks without imposing new elements to the network. Through a comparison with other mobility mechanisms, we show in the analytic analysis that our hybrid scheme presents better results in terms of handover latency and packet loss.
\end{abstract}

\section{KEYWORDS}

Heterogeneous networks, IMS, MIH, vertical handover

\section{INTRODUCTION}

IMS is a framework for delivering multimedia services over IP networks. It was originally designed by the third Generation Partnership Project (3GPP) as a part of the vision of evolving mobile networks beyond GSM. IMS [1] is the new approach adopted by 3GPP towards networks convergence; it was designed to be access independent and ubiquitous.

3GPP adopted the IETF SIP standard for IMS signaling which is considered by nature compatible with internet world. IMS architecture is organized in three horizontal layers that separate user plane, control plane and application plane (see Figure 1). This layering scheme allows isolating the service from network layer by interjecting session control functions for all application types. Therefore any operator can offer a unified interface to service providers to develop a wide range of applications independently. Nevertheless, mobility management through heterogeneous networks is a new challenge that IMS should face. IMS already provides personal mobility for nomadic users, but still need to deal with service continuity within non 3GPP networks. Indeed, IMS inherits mobile faculties from SIP, however, terminal mobility performance is questionable especially for mid session mobility in heterogeneous networks [2].

DOI : 10.5121/ijenc.2011.3311 
For this reason, 3GPP has integrated only personal mobility into IMS framework and defined different scenarios for session continuity according to the degree of session control.

In order to overcome SIP limitations in providing seamless service continuity, another mobility management protocol (MMP) should take over the handover management completely or partially to reduce handover latency as well as the number of lost packets. Many mechanisms were proposed in the literature to provide an alternative to SIP or supporting it in achieving a seamless handover. Nevertheless, these mechanisms have themselves some limitations or inflict a big change to the network. In this paper we propose a new hybrid scheme integrating two mobility solutions with minimum changes in the network. We consider two scenarios: the first one is called selected handover and the second one is called forced handover. Selected handover takes place when the mobile detects a new network using an interface other than the one currently receiving the stream. Although the mobile does not experience any noticeable degradation in the old network, a new interface gets connected to a network that presents better QoS parameters. Therefore, UE decides to switch the flow from the current interface to the new one. Whereas forced handover is performed where a multi interface UE is obliged to change its point of attachment because of signal fading or QoS degradation. Pre-established thresholds determine the minimal level of signal power required to maintain user experience undisrupted. Under these conditions, our purpose is to minimize handover latency and packet loss with minimal changes in the network. Forced handover consists of two phases; the first phase uses an enhanced FMIPv6 [3] to preserve packets from loss during layer 2 handoff. A tunnel is created between the two access routers in order to forward data packets and buffer them in the new access router. As soon as the UE connects to the new access network, data packets are forwarded to UE on the new link. The second phase uses SIP to update the session and redirect packets to the new destination. We show in the analytical study that the handover performance is improved.

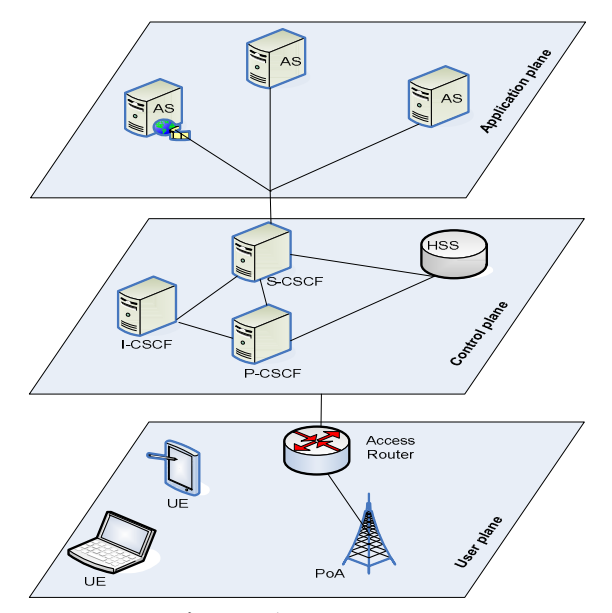

Figure 1: IMS layers

\section{RELATED WORK}

This section gives an overview of IMS architecture and the interworking methods between heterogeneous networks in IMS. Afterwards, some related works to mobility management in IMS are presented and discussed.

\subsection{IMS Architecture}

IMS architecture introduces new elements to establish and control multimedia session. The main components of IMS are the following: 
- Proxy-Call Session Control Function (P-CSCF) is considered as the interface between UE and IMS network in order to redirect SIP messages between IMS network and UE.

- Interrogating-Call Session Control Function (I-CSCF) is a SIP proxy server which routes SIP request to the adequate S-CSCF.

- Serving-Call Session Control Function (S-CSCF) is the central point of the IMS control plane. It acts as a SIP registrar which maintains a binding between the user location and the user SIP address.

- Application Server (AS) hosts and executes all services offered by IMS.

- Home Subscriber Server (HSS) dose a similar function as HLR (Home Location Register) in the GSM network. This means that it is a database of all subscribers' profiles.

\subsection{Interworking}

Interworking is the Integration of several heterogeneous access networks. In the context of IMS, interworking is attaching all access networks to the IMS core (see Figure 2). This attachment is done either physically or logically in order to control the session regardless the access network from which UE is connected. There are two levels of integration:

- Tight coupled interworking: also known as 3GPP IP access mode, in this case the WLAN/WMAN is attached physically or via a tunnel to the core network and is totally controlled by the 3GPP network. Two types of IP addresses are necessary in this case. The first one is allocated by the WLAN network and the second one is allocated by the 3GPP network. An IPsec tunnel is established between UE and packet data gateway (PDG) which is placed in the entry of the $3 \mathrm{GPP}$ network and receives all the traffic coming from the WLAN.

- Loosely coupled interworking: or IP direct access, in this mode only high level components especially control and signaling information are exchanged between the two networks, i.e. AAA authentication, mobility management, QoS management...etc.

$3 \mathrm{GPP}$ has detailed functional incremental scenarios to achieve the ultimate goal, which is a 3GPP subscriber with a WLAN radio interface having the same services as when using a 3 GPP radio interface (GSM, GPRS, UMTS) with mobility between 3GPP and non-3GPP radio interfaces similar to intra-3GPP mobility. The six scenarios that 3GPP has defined correspond to incremental interworking functionalities from 1 to 6 as shown in Table 1.

Table 1: incremental interworking scenarios

\begin{tabular}{|c|c|c|c|c|c|c|}
\hline & $\begin{array}{c}\text { Scenario 1: } \\
\text { Common } \\
\text { billing and } \\
\text { customer } \\
\text { care }\end{array}$ & $\begin{array}{c}\text { Scenario 2: } \\
\text { 3GPP } \\
\text { system } \\
\text { based } \\
\text { access } \\
\text { control and } \\
\text { charging }\end{array}$ & $\begin{array}{c}\text { Scenario 3: } \\
\text { Access to } \\
\text { 3GPP } \\
\text { system PS } \\
\text { based } \\
\text { services } \\
\text { Capabilities: }\end{array}$ & $\begin{array}{c}\text { Scenario 4: } \\
\text { Service } \\
\text { continuity }\end{array}$ & $\begin{array}{c}\text { Scenario 5: } \\
\text { Seamless } \\
\text { services }\end{array}$ & $\begin{array}{c}\text { Scenario 6: } \\
\text { Access to } \\
\text { 3GPP } \\
\text { system CS } \\
\text { based } \\
\text { services }\end{array}$ \\
\hline Common billing & $X$ & $X$ & $X$ & $X$ & $X$ & $X$ \\
\hline $\begin{array}{c}\text { Common customer care } \\
\text { 3GPP system based } \\
\text { access control }\end{array}$ & $X$ & $X$ & $X$ & $X$ & $X$ & $X$ \\
\hline $\begin{array}{c}\text { 3GPP system based } \\
\text { access charging }\end{array}$ & & $X$ & $X$ & $X$ & $X$ & $X$ \\
\hline $\begin{array}{c}\text { Access to 3GPP system } \\
\text { PS based services from } \\
\text { WLAN }\end{array}$ & & $X$ & $X$ & $X$ & $X$ & $X$ \\
\hline $\begin{array}{c}\text { Service continuity } \\
\text { Seamless service } \\
\text { continuity }\end{array}$ & & & & $X$ & $X$ & $X$ \\
\hline $\begin{array}{c}\text { Access to 3GPP system } \\
\text { CS based services with } \\
\text { seamless mobility }\end{array}$ & & & & & $X$ & $X$ \\
\hline
\end{tabular}

For each scenario there are additional functionalities that should be added to the architecture to support the aimed service. 


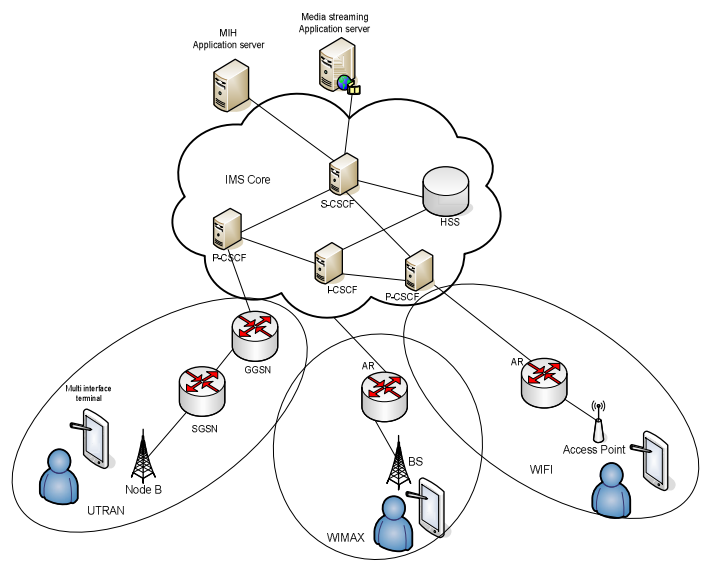

Figure 2: Interworking architecture in IMS

Interworking is the first step towards service continuity in access independent IMS. [4] specifies system description for interworking between 3GPP and WLANs. As for WIMAX interworking with 3GPP, WIMAX forum has proposed an interworking architecture [5] based on the same documents as WLAN interworking. It covers both direct IP access (loosely coupled) and 3GPP IP access (tightly coupled), it does not change the previous one, but only add, the missing elements for WIMAX interworking.

\subsection{Mobility within IMS}

In [6] three mobility management protocols (MIPv6, SIP, and PMIPv6) are investigated in IMS 3GPP2 context for heterogeneous access networks (i.e. WIFI, CDMA2000). The experimental results show a handover delay of up to 4s for MIPv6 and PMIPv6. This delay reaches 9s when SIP mobility is used.

In [7] the authors use MIP to handle mobility of TCP traffic, and SIP for real time traffic. MIP and SIP create a kind of redundancy in the network and cause double registration and double binding update. Although the authors of [8] tried to reduce this redundancy by merging entities that have similar functionality, still traffic separation is used which adds more complexity in the network. Moreover using only SIP for real time traffic in IMS framework will introduce a big handover delay.

In [10] MIP and SIP are used for real time traffic consecutively. MIP is used first to reroute the traffic to the new network and than SIP updates the route from the correspondent node by reregistering and re-inviting the session. Here again network entities are redundant due to double use of mobility protocols i.e. Home Agent (HA), Foreign Agent (FA) and sip proxy server. Moreover MIP is known to be unsuitable for real-time applications because of its triangular routing and high handover delay. The corresponding UE will update its destination IP address after receiving a re-invite message which cancels the function of HA. Netcape [11] also tries to optimize handover delay by using MIP for underlying traffic redirection.

IHMAS [12] is a proactive solution that aims to reduce vertical handoff latency; it predicts vertical handoffs at client side and starts session reconfiguration before handoff management. This proposal adopts an application layer proxy-based approach, for session signaling and data handoff. It uses application server for session continuity that reduces handoff media losses by decoupling session rebinding and data transfer times. Nevertheless, this solution takes into account only the case of make-before-break, in other words the authors assume that there is always enough time for the UE to prepare the connection to the new network before leaving the original one. While SIP operations (register and invite) in addition to the basic configuration operations need a long time to be achieved.

Media-independent pre-authentication (MPA) is a framework that allows UE to pre-authenticate proactively before layer 2 handover. It allows also a pre-configuration of UE with the 
appropriate IP address. In addition, the binding update of the corresponding network entities (HA, CN, SIP server etc) is carried out proactively whatever the used MMP. After a handover implying layer 3 modification, UE has to re-register before resuming its communications. Updating the route with SIP proactively has a good impact on handover delay and packet loss, nevertheless, the long time taken by SIP to re-register and update the session is not always easy to predict. Actually, handover predication mechanisms, especially layer 2 triggers such as link going down and link down are the key elements for a successful proactive handover. On the one hand the whole handover procedure is triggered by a warning event (i.e link going down) based on signal strength or QoS degradation. On the other hand, the time between this first warning and the instant of the actual disconnection or QoS degradation notified by link down event is beyond user control. The remaining connection time should be at least equal to all time needed by the mobile to perform successful proactive update. This time is remarkably high in the case of SIP, given the multitude of network elements intervening in re-registration procedure. The handover preparation may go in vain because of unpredicted disconnection; hence the mobile has to update its session reactively once connected to the new network.

\section{HYBRID MECHANISM}

In this section we will present our solution for IMS mobility using enhanced FMIPv6 and SIP.

\subsection{MIH integration to IMS framework}

Media independent handover [9] aims to provide MMPs with intelligence in executing both vertical and horizontal handover. Integrating MIH to IMS is done at both access network and core network. In the access network, MIH is implemented in UE, PoA and AR (or GGSN); whereas the information server (IS) is placed in the core network.

As far as media independent information service (MIIS) is concerned, the communication between IS and the remote MIHF is organized following a request response paradigm. In [13], HTTP is used to retrieve the needed information from the IS. The authors of [14] propose SIP to transport MIIS messages, but they don't specify how this information is transported neither the adequate SIP messages to carry them. Actually, SIP seems to be a good solution for transporting MIIS messages and will facilitate the integration of IS into IMS architecture. We propose to deploy IS as an application server (AS) placed in the core network in order to be accessed from all access networks by authenticated and authorized users. IS has a database of information about access networks. Examples of such information are: geographical location, operator name, PoA MAC address, IP address prefix of its AR, operational channel...etc.

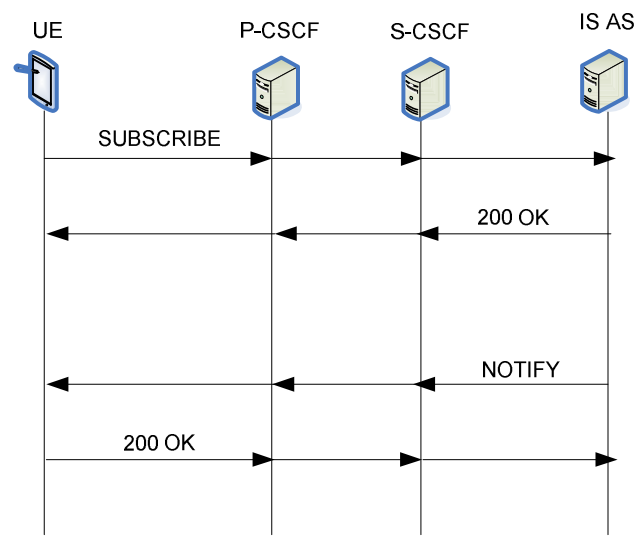

Figure 3: IS integration into IMS

Surrounding networks of a given UE can be determined using its IP address or its GPS coordinates if available. Access to this information is conditioned by the authorization of the 
HSS which allows only users who are already subscribed to this service to reach the needed information. Moreover, the need for neighboring access network knowledge is done at least once after being connected to the network. Updates of the list of neighboring networks can be done in a regular basis depending on UE mobility behavior.

After address configuration and re-registration if necessary, UE should learn about the available wireless networks in its area in order to have a list of candidate networks to handover to in case the signal of the current connection goes down. This anticipation will be very benefic for UE when it needs to reverse resolve the IP address of the AR behind the discovered PoA. Indeed this resolution will be done locally when the handover is imminent, thus reducing the handover preparation delay. In the context of IMS, the list of the neighboring networks will be provided using SUBSCRIBE and NOTIFY messages. It should be noted here that MIH standard does not specify any transport mechanism for information service messages. We propose to carry the info request message on SDP payload of Subscribe and the info response message on notify SDP payload as illustrated in Figure 3.

\subsection{Hybrid scheme for IMS mobility}

The goal of any handover scheme is to ensure service continuity with an acceptable perceived quality of experience for the user during the transition. We classify mobility operations over IP into four main operations: movement detection and address allocation, global location tracking, traffic redirection and handover smoothing. SIP can perform the three first operations by nature. The missing part towards seamless service continuity is the handover smoothing. The idea behind our proposition is to propose a solution based on MIH intelligence to make an appropriate anticipation operation depending on low layers events. Our solution does not imply a big change to the existing network and avoids redundancy among the intervening entities. We will use an enhanced version of FMIPv6 combined with SIP as mobility management protocols. We consider intra domain inter technology and intra domain intra technology handovers. This means that the whole network including all access networks are controlled by the same administrative authority and the mobile can travel among access networks within the same or different technologies (e.g. WIMAX to WIMAX or WIMAX to UMTS). Access networks are connected to IMS core network either loosely or tightly. The advantage of using FMIPv6 is that there is no need for home agent since the only mission of FMIPv6 is to preserve the current session by forwarding and buffering packets from the old AR to the new AR respectively. In other words, FMIPv6 will take in charge the handover smoothing operation, whereas global location tracking and traffic redirection will be performed by SIP.

\subsubsection{Selected handover}

In selected handover, there is no time constraint on handover achievement since the UE continues in receiving the traffic from the current interface and prepares at the same time all needed updates using the new interface. Special care should be taken in the triggering mechanism of such handover. It is even the major criterion that decides on what type of handover has to be carried out. Therefore, basing on the MIH framework, the adequate triggers that should lead to selected handover are of two types: link up and link detected. After notification of the MIHF, decision algorithm is executed and decides whether the new network is better or not. Obviously, UE would prefer to be always best connected, therefore when a better network is detected by any other interface, decision engine compares the offered quality of the new network with the quality of the current network. By the term "quality" we refer to a group of criterions that have been configured in the decision engine and should be taken into account when deciding which network is better. Those criterions can include bandwidth, cost, user preferences... etc. UE performs SIP related updates before switching to the new network. It should be noted that always the first operation is IP address acquisition. This operation can be performed by means of different methods: stateful method (e.g DHCP, PDP) or a stateless method (e.g autoconfiguration). IP address acquisition is done normally like if the UE intends to connect to the network using this interface for the first time without taking into account the 
ongoing handover. After that, UE starts updating the session. First of all, it should discover the new P-CSCF as it could differ from the old one. An additional optimization in the handover that can be performed using MIH framework is that the information about the new P-CSCF can be provided in the info response that was acquired earlier. Afterwards UE negotiates a security association with the P-CSCF in order to construct an IPsec tunnel to secure communications between UE and P-CSCF. Subsequently, UE starts updating the session by re-registering to the S-CSCF which needs first to re-authenticate UE since it is using a different IP address now. Dual use of IP address and being registered to IMS domain with both of them an allowed operation which is known as multiple registrations. Until now traffic is following the old route towards the old IP address since the interface is still connected. Eventually, UE sends re-invite to all its corresponding UEs in order to update the destination address of their streams towards the mobile UE. Finally this latter UE can deregister the previous IP address by sending deregister message from the old interface. Message flow corresponding to selected handover scenario is shown in Figure 4. Message exchange related to IP configuration, security association and I-CSCF/HSS are omitted.

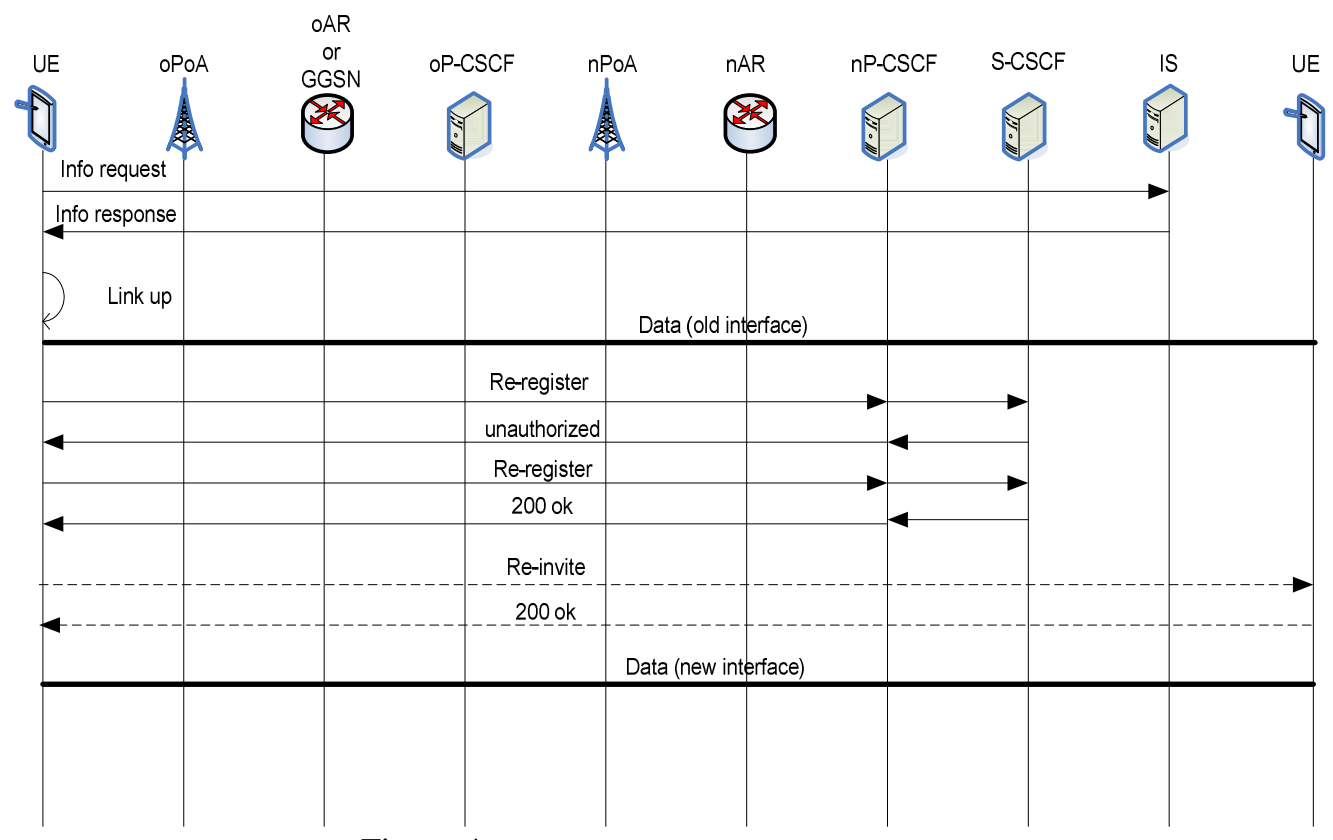

Figure 4: Selected handover message exchange

\subsubsection{Forced handover}

Forced handover flow chart is shown in Figure 5. In this scenario UE is constrained to change its connection to a new network using the current interface or a second one which is not already connected. The mechanism is carried out in two phases: fast phase and slow phase. 


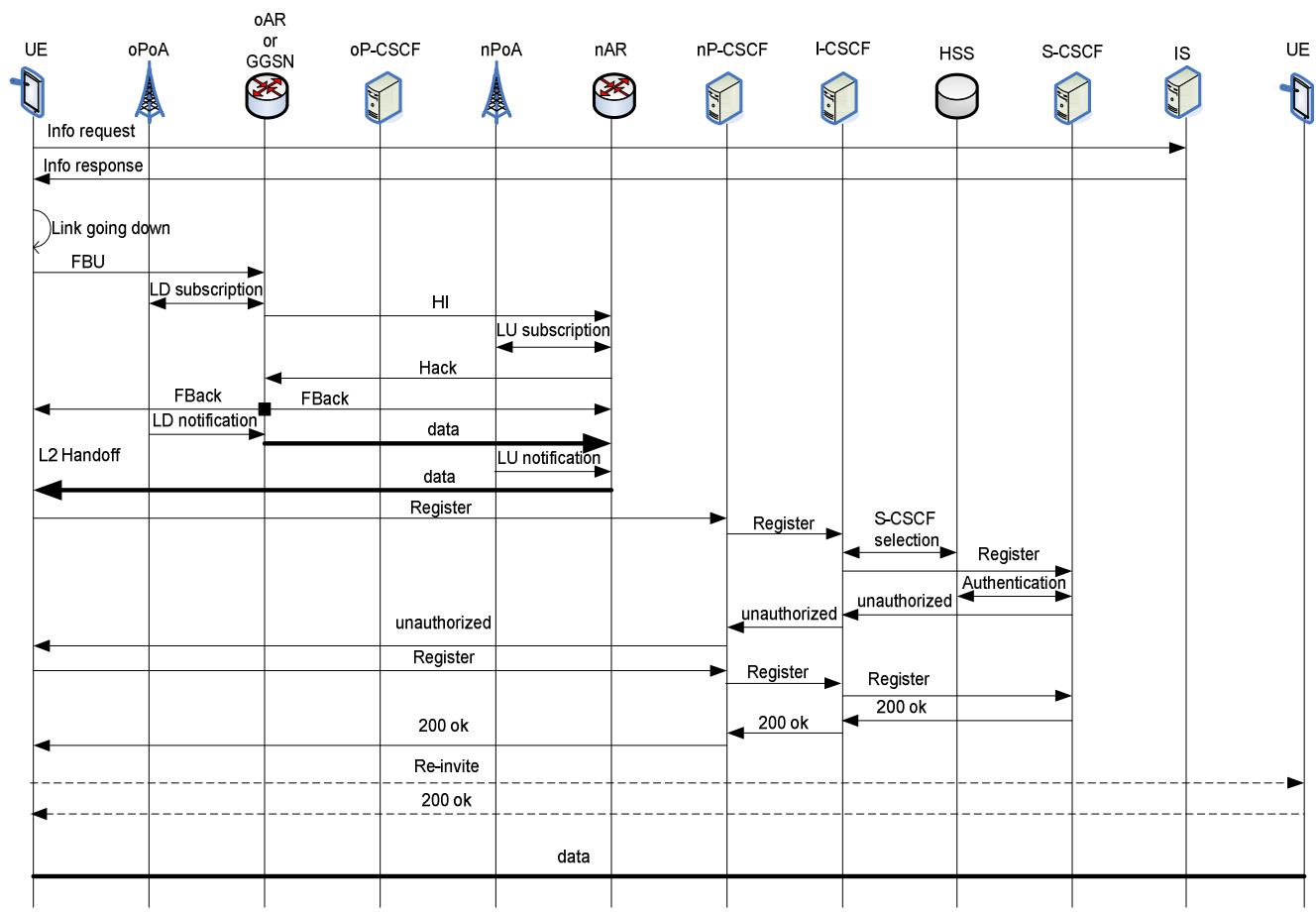

Figure 5: The proposed hybrid handover mechanism

\section{A. Fast phase}

Our first priority is to preserve packets of the ongoing session before any other procedure. The first operation is the reverse resolution of the new PoA mac address to acquire the new access router (NAR) prefix. UE uses IS to know about the neighboring networks and their AR prefixes before the handover trigger. After a link going down trigger, UE scans the channels of its interfaces and resolves the NAR address locally using the information provided in the info response of the IS and then constructs a Prospective CoA. After that, FMIPv6 operation is carried out to protect packets from loss. Nevertheless, sending a fast binding update (FBU) message will prevent UE from receiving any packet because they are encapsulated to the NAR. So we need to trigger the tunnel creation by another mechanism which is separated from FBU transmission. In a previous work [16], we proposed a collaborative scheme to achieve seamless vertical handovers using MIH services efficiently in both terminal side and network side. This scheme allows the terminal continuing its communication in the previous network even after link going down notification. The tunnel between the two ARs allows preservation of data packets during layer 2 handover and continuity of the service while performing SIP updates.

\section{B. Slow phase}

As soon as UE connects to the new access network, it starts updating its SIP session while receiving data packets through the tunnel. It should be noted here that SIP related messages are sent with the new IP address, whereas data packets are encapsulated using the old IP address and sent through the tunnel. The reason behind this distinction is that the corresponding UE may drop data packets if they are sent with a source address witch is different from the one that the session was established with in the first place. Only after receiving re-invite message, correspondent UE takes into account the new destination address. On the contrary, SIP messages should be sent directly without encapsulation because this address will be used in security association establishment between UE and the new P-CSCF.

UE starts by discovering its new P-CSCF. This information can also be provided by the IS in the previous information response. Afterwards, UE is re-authenticated, re-registered and finally re-invites the corresponding UE. 
Note that this scenario is based on the assumption that FMIPv6 predictive mode is successful. Indeed, FMIPv6 Predictive mode has more chance to succeed than SIP update anticipation, because it only needs to send FBU message. Whereas, the success of SIP session update requires success of several operations. In the case where FBU is not received in the old link, the reactive mode is activated and FBU is sent from the new network.

\subsection{TCP and UDP traffic support}

For this hybrid scheme to work well, long sessions like voice calls and multimedia streaming should be transported over a connectionless channel like UDP. For text based web access, traffic model [15] does not require service continuity since it is an on/off behavior (download and read). But for long TCP connections as in the case of downloading big files using FTP, FMIPv6 tunnel should be kept up until the end of the download. Although FTP sessions inflict a long tunnel establishment time, this scheme is better than MIP where the tunnel is permanent regardless the application currently in use. In FMIPv6, The information about the tunnel establishment time is inserted by the UE in FBU message and transmitted to the NAR by the HI message. The difficulty of this solution resides in the estimation of the remaining TCP connection time and the mechanism to maintain the tunnel established along this period. Actually we need to modify FMIPv6 protocol to add a new mechanism for maintaining and terminating the tunnel between PAR and NAR.

In case of selected handover, the solution is simpler since it suffices that the decision engine postpones the handover execution until all TCP connections are closed. In such cross layer design, the decision engine is in the center of information flows coming from lower layers (i.e. link events) and upper layer (i.e. TCP/UDP session).

\section{PERFORMANCE EVALUATION}

In this section performance of our mobility management scheme is analyzed and compared with SIP and MIP-SIP solutions. The studied performance parameters are handover delay and packet loss.

\subsection{Handover latency}

In a multimedia entertainment, the handover delay should be reduced to its minimum so that users do not feel any interruption in the session during the handover. The majority of the media are transported via RTP/UDP where no retransmission is available. Therefore, any lost packet will penalize users' quality of experience.

Handover delay or latency is defined as the time separating the reception of the last packet in the old network and the reception of the first packet in the new network. The total handover delay $D_{H}$ is the addition of 3 components:

$$
D_{H}=D_{H 2}+D_{L 3}+D_{M M P}
$$

Where $D_{H 2}$ is layer 2 handover delay. We assume equal values of $D_{H 2}$ for both vertical and horizontal handovers since in this scenario the new interface is not up at the time of handover decision.

Table 2: Notation table

\begin{tabular}{|l|l|}
\hline$D_{U E-A R}$ & Delay between UE and AR \\
\hline$D_{U E-P}$ & Delay between UE and P-CSCF \\
\hline$D_{P-I}$ & Delay between P-CSCF and I-CSCF \\
\hline$D_{I-S}$ & Delay between I-CSCF and S-CSCF \\
\hline$D_{S-H}$ & Delay between S-CSCF and HSS \\
\hline$D_{P-S}$ & Delay between P-CSCF and S-CSCF \\
\hline$D_{U E-H A}$ & Delay between UE and HA \\
\hline$D_{P O A-A R}$ & Delay between PoA and AR \\
\hline
\end{tabular}


$D_{L 3}$ is the delay of layer 3 operations; it concerns mainly IP address acquisition

$D_{M M P}$ is the delay inflicted by the MMP to update the related entities following the used MMP. The rest of the notations are reported in Table 2 .

\section{A. SIP}

UE acquires the IP address using neighbor discovery protocol, which consists of sending a solicitation to the AR and receiving an advertisement. Then it discovers the new P-CSCF, registers with the S-CSCF and re-invites the corresponding UE which corresponds to the delays $D_{\text {disc }}, D_{\text {regis }} D_{i n v}$ respectively. In case of using SIP as MMP we have:

$$
\begin{aligned}
D_{L 3} & =D_{s o l}+D_{a d v} \\
& =2 D_{U E-A R}
\end{aligned}
$$

We can write $D_{S I P}$ as:

$$
D_{S I P}=D_{\text {disc }}+D_{\text {regis }}+D_{\text {inv }}
$$

with

$$
\begin{aligned}
D_{d i s c} & =D_{U E-P}+D_{P-U E} \\
& =2 D_{U E-P}
\end{aligned}
$$

and

and

$$
D_{\text {regist }}=4 D_{U E-P}+4 D_{P-I}+4 D_{I-S}+2 D_{I-H}+2 D_{S-H}
$$

$$
D_{i n v}=4 D_{U E-P}+4 D_{P-S}
$$

We suppose that all paths are symmetric and have the same transmission delay in both directions.

\section{B. MIP-SIP}

In MIPv6 the CoA is first acquired from the AR and then binding update is performed within HA. Subsequently packets are forwarded by HA to UE. In MIP-SIP scheme we have:

\section{E-FMIP-SIP}

$$
\begin{aligned}
D_{L 3} & =D_{s o l}+D_{a d v} \\
D_{M I P} & =D_{B U}+D_{a c k} \\
& =2 D_{U E-A R}+2 D_{U E-H A}
\end{aligned}
$$

The advantage of using FMIPv6 is that the IP address acquisition is done proactively. So, $D_{L 3}$ is null in this case.

Upon detecting a link down in the PoA packets are forwarded through a tunnel between PAR and NAR, and buffered at NAR simultaneously with layer 2 handoff. Once NAR is notified of a link up, packets are delivered. Thus in predictive mode with MIH enhancement (E-FMIP) we have:

$$
\begin{aligned}
D_{E-F M I P} & =D_{L U n o t}+D_{A R-U E} \\
& =D_{P o A-A R}+D_{A R-U E}
\end{aligned}
$$

as for reactive mode

$$
\begin{aligned}
D_{F M I P r e a} & =D_{U N A}+D_{F B U}+D_{H I}+D_{H a c k}+D_{F B a c k} \\
& =3 D_{U E-A R}+4 D_{A R-A R}
\end{aligned}
$$




\subsection{Numerical results}

\subsubsection{Handover delay}

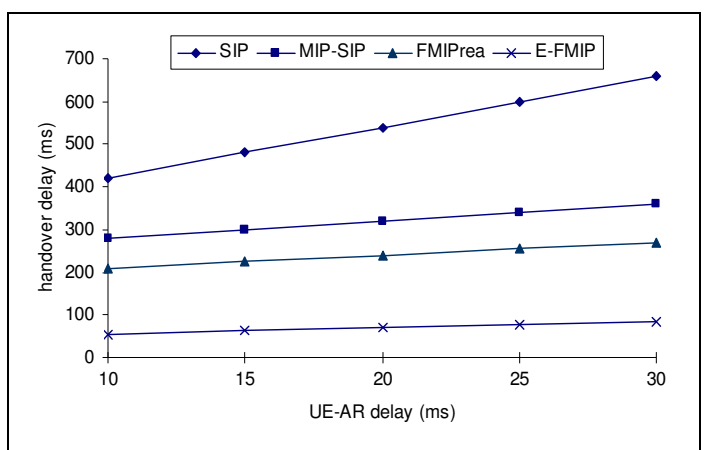

Figure 6: Handover delay vs UE-AR delay

Figure 6 shows that the handover delay of the E-FMIPv6 scheme is low compared to the other schemes even when the access link delay is high which corresponds to complicated access network architecture like GPRS. Nevertheless success of the predictive mode is crucial to perform such low delay. As depicted in Figure 7, FMIPv6 scheme is not sensible to the distance that separates UE from its home network since it involves only the AR of the concerned networks. It also reduces the triangular routing to a small scale.

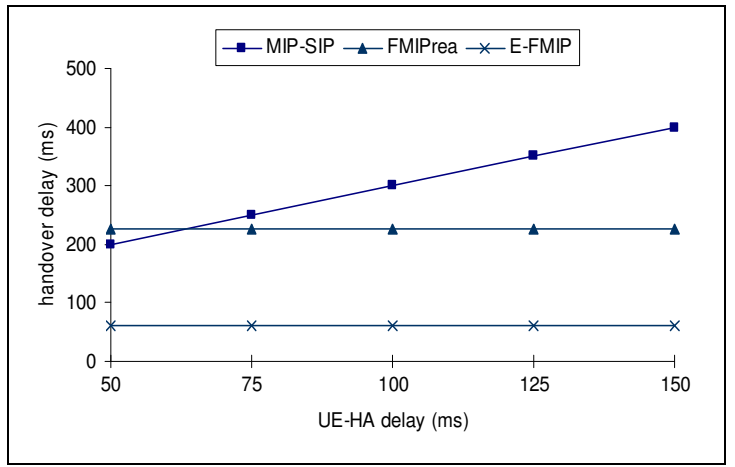

Figure 7: Handover delay vs UE-HA delay

\subsubsection{Packet loss}

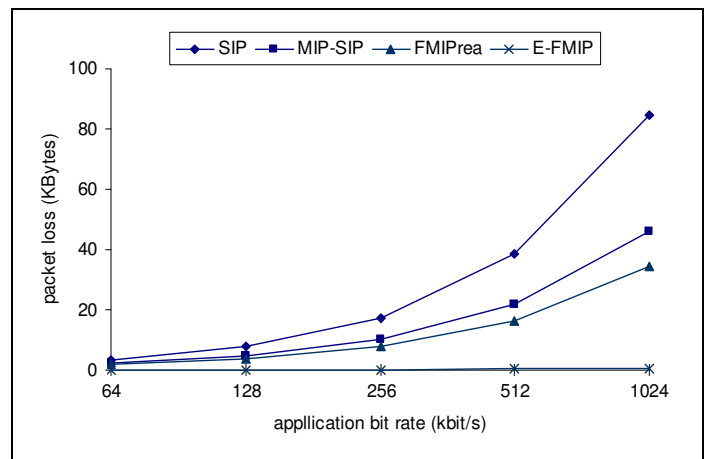

Figure 8: Packet loss vs application bit rate

During the handover some packets may be lost if they are not buffered as in FMIPv6. The amount of lost data is expressed as follows: 


$$
\mathrm{PL}=\int_{0}^{D_{H}} R\left(T_{0}+t\right) d t
$$

Where $\mathrm{R}(\mathrm{t})$ is the application bit rate and $T_{0}$ is the instant of the last received packet on the old link. Figure 8 shows that packet loss in our solution is nearly null. More concretely, in a video streaming application that has a bit rate of $1024 \mathrm{kbit} / \mathrm{s}$ there are only one or two packets which are lost during the handover. Such a weak number of lost packets does not have a big impact on the perceived quality. On the contrary, the user will miss a long video sequence during the handover when the other MMPs are used.

\section{CONCLUSION}

Interworking between different access networks is an important step towards networks convergence. But IMS mobility over heterogeneous access networks is still an open issue. In order to offer ubiquitous service to $4 \mathrm{G}$ customers, telecom operators should resolve vertical handover issue. In this paper we proposed a hybrid scheme using enhanced FMIPv6 and SIP. This scheme does not impose any new entities on the network as MIPv6 does. A performance evaluation of the proposed solution was carried out and compared to the other schemes. The results show that our hybrid scheme reduces the handover delay and packet loss. However, these results are tightly dependant on the success of the predictive mode of FMIPv6. Our future work will focus on the issue of TCP connections. Special protocols have to be designed in order to control tunnel establishment regarding the nature of the ongoing sessions.

\section{REFERENCES}

[1] 3GPP, "IP Multimedia Subsystem (IMS); stage 2 (Release 9)” TS23.228 V9.0.0, Dec. 2009

[2] Dutta, A.; et al, "A-IMS architecture analysis and experimental IPv6 testbed" International Conference on IP Multimedia Subsystem Architecture and Applications, 2007

[3] R. Koodli, Ed., "Fast Handovers for Mobile IPv6”, RFC 5568, IETF Network Working Group, July 2009

[4] 3GPP, “3GPP system to Wireless Local Area Network (WLAN) interworking,stage 2 (Release 9)” TS23.234 V9.0.0, Dec. 2009

[5] WIMAX forum "WiMAX - 3GPP Interworking”, WMF-T37-002-R010v3. Nov 2008

[6] Chiba, T.; et al; "Performance Analysis of Next Generation Mobility Protocols for IMS/MMD Networks" IWCMC '08.6-8 Aug. 2008 .pp. 68 - 73

[7] Wong K., et al,"A Multilayered Mobility Management Scheme for Auto-Configured Wireless IP Networks". IEEE Wireless Communications, vol. 10, no. 5,pp. 62 - 69. Oct 2003.

[8] Wang Q. and Abu-Rgheff M. "Mobility Management Architectures based on Joint Mobile IP and SIP Protocols". IEEE Wireless Communications, vol. 13, pp 68-76, Dec 2006

[9] IEEE Standard for local and Metropolitan Area Networks, Part 21: Media Independent Handover Services", January 2009

[10] Munasinghe, K.S.; Jamalipour, A.: “A Unified Mobility and Session Management Platform for Next Generation Mobile Networks”, IEEE GLOBECOM '07. pp 4979 - 4983

[11] A. Udugama et al., "NetCAPE: Enabling Seamless IMS Service Delivery across Heterogeneous Mobile Networks,’IEEE Commun. Mag., vol. 45, no. 7, July 2007,pp. 84-91.

[12] Bellavista, P., Corradi, A., Foschini, L., "IMS-Compliant management of vertical handoffs for mobile multimedia session continuity"IEEE Commun. Mag., vol. 48, no. 4, April 2010, 114 121

[13] Dutta, et al, "An Experimental Study of Location Assisted Proactive Handover ", IEEE GLOBECOM, November 2007, Washington. 
International Journal of Computer Networks \& Communications (IJCNC) Vol.3, No.3, May 2011

[14] Silvana, G.P.; Schulzrinne, H.; "SIP and 802.21 for Service Mobility and Pro-active Authentication", 6th Annual Communication Networks and Services Research Conference, 2008. pp: $176-182$

[15] Hyoung-Kee Choi, Limb, J.O., "A behavioral model of Web traffic" Seventh International Conference on Network Protocols, 1999. (ICNP '99) pp 327 - 334

[16] M. BOUTABIA, H. Afifi "collaborative handover mechanism for real time services", The 9th International Conference on ITS Telecommunications 2009. October 20-22 Lille France 